\title{
Study on Scatter of Surface Integrity of Bearing Raceway Grinding
}

\author{
Zhou Chang (0000-0002-2619-0290) ${ }^{1}$, Lai Hu (0000-0002-7715-5612) ${ }^{2}$ \\ ${ }^{1}$ School of mechanical and electrical engineering, Lanzhou Jiaotong University, Lanzhou, Gansu, P.R. China \\ 730070 E-mail: starismyfriend@163.com \\ 2State Key Laboratory for Manufacturing System Engineering, Xi'an Jiaotong University, Xi'an, Shaanxi, P.R. \\ China, 710054
}

Taking the discrete degree of bearing raceway grinding surface integrity as the research object, the orthogonal test based on the wheel speed, workpiece speed and grinding depth is designed. The residual stress, retained austenite, hardness, grinding modified layer thickness and roughness of the raceway after grinding test were measured, and the mean value and standard deviation of these indexes were calculated. The results show that the standard deviation of residual stress and roughness decreases with the increase of wheel speed, and the standard deviation of retained austenite, hardness and grinding modified layer thickness keeps stable. Finally, the optimal grinding parameters considering the scatter of surface integrity are selected.

Keywords: raceway grinding; surface integrity; orthogonal test; scatter; standard deviation

\section{Introduction}

Bearing is an important mechanical functional component. The raceway is the working surface of the bearing, and its performance usually affects the service condition of the bearing. Grinding is usually used as an important finishing method for bearing raceway. The grinding process of bearing raceway directly affects the performance of bearing raceway. Therefore, it is very important to improve the grinding technology of bearing raceway.

Guo (GUO Y, 2009) proposed a theory that the surface integrity of bearing raceway is an important index to evaluate grinding technology. The surface integrity of bearing raceway includes two parts: surface texture and surface physical properties. Surface texture refers to the geometric accuracy of the bearing raceway, and the main parameters are the roughness of the bearing raceway. The surface physical properties include residual stress, hardness, metallographic structure, grinding modified layer, etc.

Yuan (YUAN Z-J, 1989) studied the surface integrity of bearing steel after grinding. The results show that the surface texture of bearing steel after grinding with CBN wheel is better, and the roughness of raceway is lower; the surface physical properties are better after grinding, and the residual stress is compressive stress. Abrāo (ABRAO A M, 1996) and König (KONIG W 1993) have compared the surface integrity of bearing steel after turning and grinding, and the results show that hard turning can also have good surface integrity. The above research can provide theoretical basis for bearing raceway grinding, and is widely used in bearing manufacturing.
Liu et al. (LIU C R, 1989) proposed the concept of residual stress scatter. Liu pointed out that due to the influence of residual stress on the fatigue life of mechanical products, we should not only pay attention to the average value of residual stress, but also pay attention to the scatter of residual stress. The research results of Liu show that the residual stress of grinding is more discrete than that of turning. At present, Gao (GAO E-W 2008) and Zhang (ZHANG X-P, 2010) continue to study the scatter of residual stress, study and compare the residual stress scatter of rough grinding and fine grinding, and study and compare the residual stress scatter of rough turning and fine turning. Dana (DANA S, 2019) studied the implementations of suitable cutting parameters by grinding of titanium VT9 with impact on surface integrity. Martin (MARTIN M, 2019) studied the impact of changes in infeed rate on surface integrity after chrome plate grinding by silicon carbide.

The discreteness of residual stress is studied above, but there is no literature on the discreteness of bearing raceway surface integrity. The discrete degree of the surface texture of the bearing raceway determines the geometric accuracy of the bearing raceway, and the discrete degree of the physical characteristics of the bearing raceway surface determines the life of the bearing raceway.

In this paper, the concept of surface integrity scatter is proposed. The research not only focuses on the scatter of residual stress in bearing raceway, but also on the scatter of all parameters related to surface integrity, such as roughness, hardness, metallographic structure, grinding modified layer, etc. In this paper, the orthogonal experiment of grinding is designed. 
Different grinding parameters are used to grind the raceway. The surface integrity of bearing raceway after grinding is studied and the variation law of surface integrity scatter is summarized. This paper has good engineering guidance significance.

\section{Grinding experiment description}

The test piece is the outer ring of the angular contact ball bearing (model: 7014ac). The outer diameter of the ferrule test piece is $110 \mathrm{~mm}$, the inner diameter is $98 \mathrm{~mm}$, the width is $20 \mathrm{~mm}$, and the raceway width is $7 \mathrm{~mm}$. The material of the ferrule is bearing steel GCr15. Bearing rings are hardened before grinding. The hardness after heat treatment is 61HRC. The machining machine tool is $3 \mathrm{mk} 1420 \mathrm{CNC}$ grinder, and the grinding wheel material is corundum, and the model is p $80 \times 10 \times 20 \mathrm{a} 80 \mathrm{kv} 60$. The grinding fluid is emulsion. The grinding wheel is on-line sharpened by a single point diamond pen dresser. After grinding the raceway of the ferrule every time, the diamond pen dresser is used to finish the grinding wheel, and the performance of the grinding wheel can be considered to be consistent. The processing equipment is shown in Fig. 1.

In the grinding process, there are many factors affecting the surface integrity of the bearing raceway, mainly including the wheel speed, workpiece speed, grinding depth, wheel characteristics, coolant characteristics, etc., among which the wheel speed, workpiece speed, grinding depth are relatively important factors affecting the surface integrity of the raceway, so the above three factors are mainly considered in this test. In order to fully consider the influence of various test factors on the surface integrity of the bearing raceway in a small number of tests, the orthogonal test method is used to determine the optimal grinding parameters. This test is a three factor and three level test problem. L9 orthogonal test table should be selected to design the test. The grinding wheel speed ns, workpiece speed and grinding depth considered in this test are discharged into the orthogonal test table, and the final arrangement scheme is shown in $\mathbf{T a b} \mathbf{1}$.

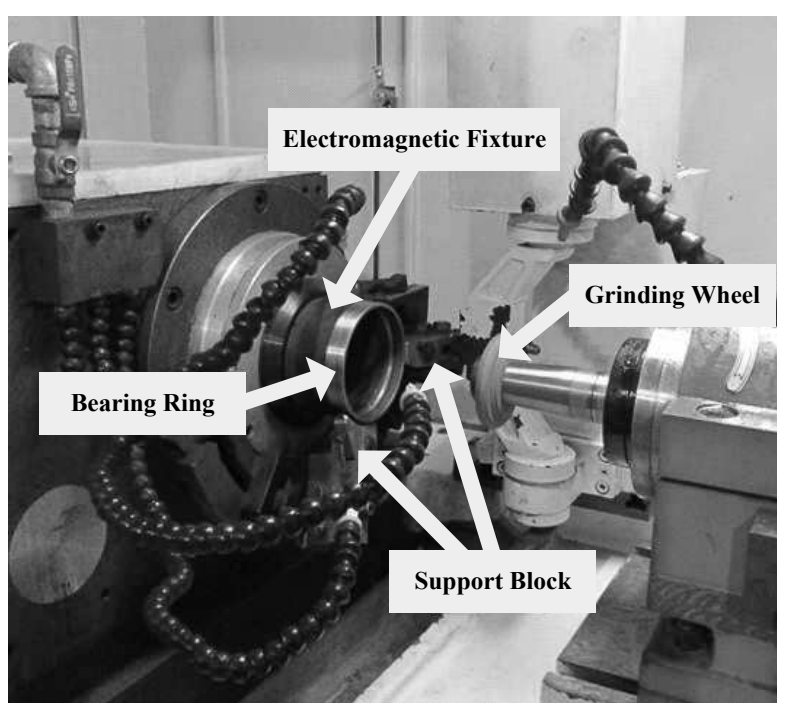

Fig. 1 The grinding equipment of bearing ring

Tab. 1 grinding orthogonal test table

\begin{tabular}{crrc}
\hline Test number & $\begin{array}{c}\text { Wheel speed } \\
\mathrm{n}_{\mathrm{s}}(\mathrm{r} / \mathrm{min})\end{array}$ & $\begin{array}{c}\text { Workpiece speed } \\
\mathrm{n}_{\mathrm{w}}(\mathrm{r} / \mathrm{min})\end{array}$ & $\begin{array}{c}\text { Grinding depth } \\
\mathrm{a}_{\mathrm{p}}(\mu \mathrm{m})\end{array}$ \\
\hline 1 & 5000 & 100 & 5 \\
2 & 5000 & 150 & 10 \\
3 & 5000 & 200 & 15 \\
4 & 7000 & 100 & 10 \\
5 & 7000 & 150 & 15 \\
6 & 7000 & 200 & 5 \\
7 & 9000 & 100 & 15 \\
8 & 9000 & 150 & 5 \\
9 & 9000 & 200 & 10 \\
\hline
\end{tabular}

\section{Test of experimental results}

There are 9 groups of grinding parameters in the orthogonal test, each group of grinding parameters is used to grind 5 rings. Each ferrule measures 3 data, a total of 135 data. For one of the indicators of surface integrity, 15 data can be obtained by using each group of grinding parameters.

Residual stress is one of the important indexes of surface integrity. In this paper, $\mathrm{x}-350 \mathrm{a} \mathrm{X}$-ray diffractometer is used to detect the residual stress. The test results are shown in Fig. 2.

It can be seen from Fig. 2 that the residual stress of the raceway after 9 groups of grinding tests is all the residual compressive stress. In the $1 \sim 6$ groups of grinding experiments, the residual pressure stress of raceway has little change, about - 200MPa. With the increase of grinding wheel speed, the residual pressure stress of raceway in $7 \sim 9$ groups of grinding experiments decreases gradually.

It can be seen from Fig. 3 that the residual stress of the raceway after 9 groups of grinding tests is both compressive stress and tensile stress. The residual stress of grinding test raceway in group $1 \sim 6$ is compressive stress, and that in group $7 \sim 9$ is tensile stress. With the increase of wheel speed, the residual stress of 
raceway changes from compressive stress to tensile stress.

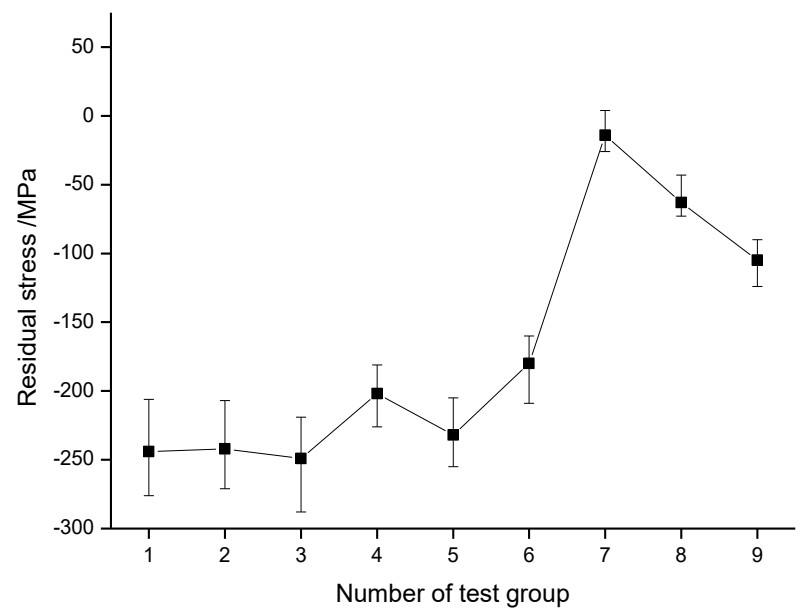

Fig. 2 Mean value of circumferential residual stress

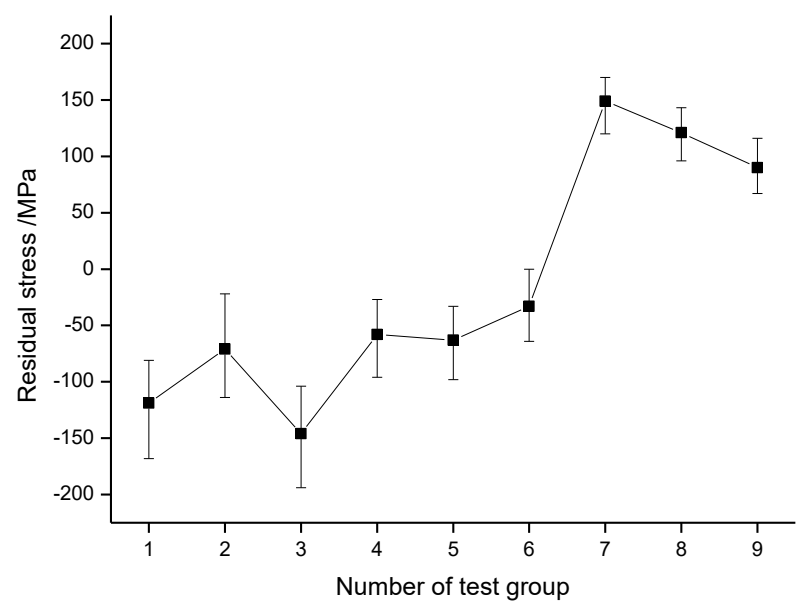

Fig. 3 Mean value of axial residual stress

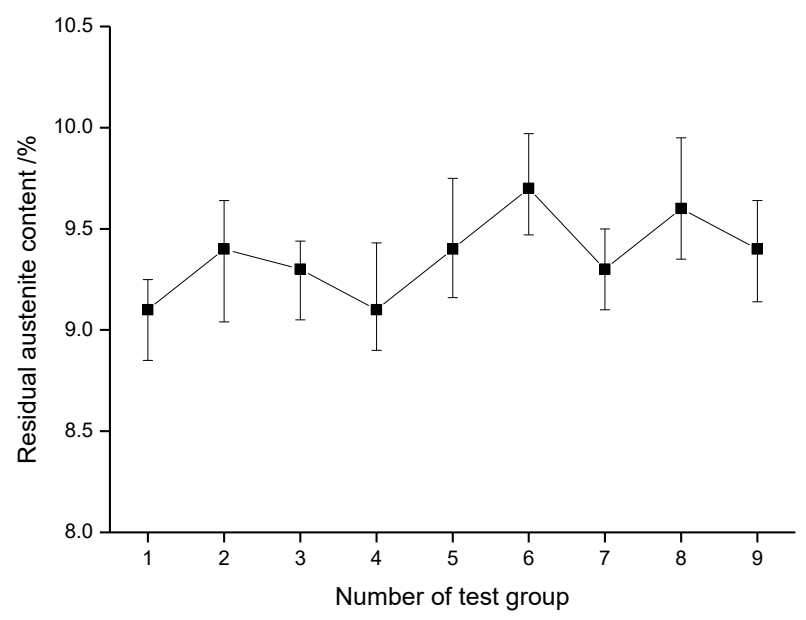

Fig. 4 Mean value of retained austenite content

The content of retained austenite in microstructure is also one of the important indexes of surface integrity. The residual austenite content can affect the bearing life. In this paper, $\mathrm{x}-350 \mathrm{a} \mathrm{X}$-ray diffractometer is used to detect retained austenite. The test results are shown in Fig. 4.

It can be seen from Fig. 4 that the content of retained austenite in the raceway after grinding is about $9 \%$, and the content of retained austenite has little change.

The hardness of raceway is one of the important indexes of surface integrity. The hardness of raceway is measured by hv- 5 , and then the Vickers hardness is converted to Rockwell hardness by hardness conversion table.

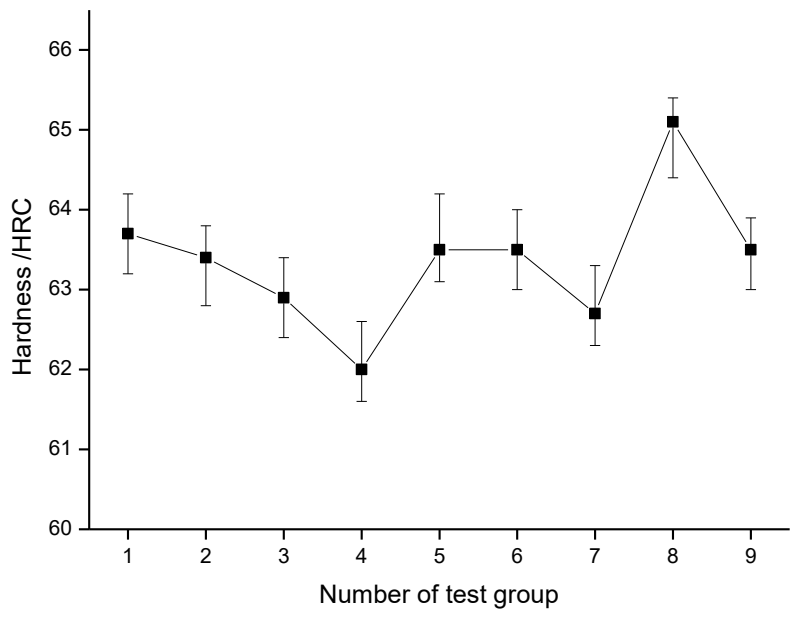

Fig. 5 Mean value of hardness

It can be seen from Fig. 5 that the hardness of the raceway after grinding is between 62HRC and 65HRC, with little change.

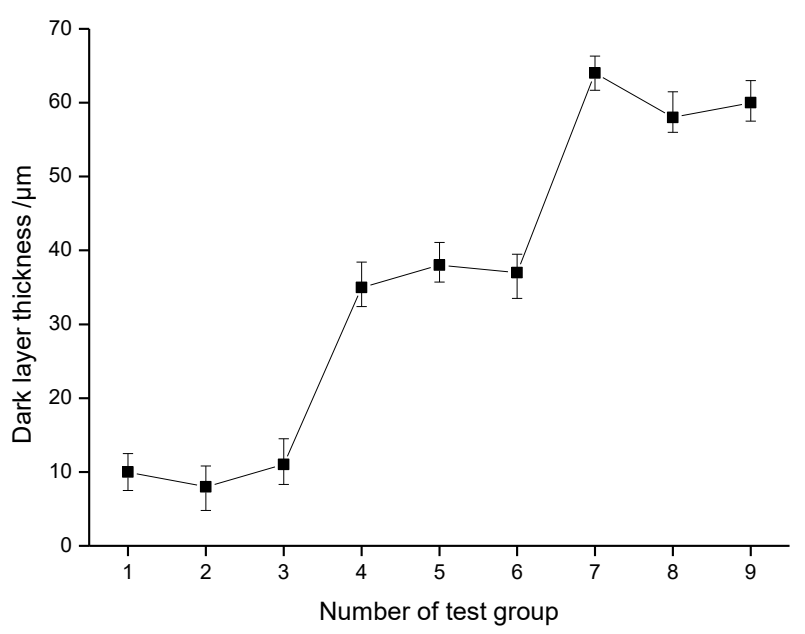

Fig. 6 Mean value of grinding modified layer thickness

The thickness of the metamorphic layer in the raceway was measured by SEM su-8010. It is specially pointed out that there is no white layer in the modified layer of 9 groups of grinding tests. It can be seen from Fig. 6 that the thickness of the dark layer in group 1 3 grinding test is about 10 microns; that in group $4 \sim 6$ grinding test is about 30 microns, and that in group 7 9 grinding test is about 50 microns.

PGI contact aspheric measuring instrument is used 
to measure the roughness of bearing raceway. The roughness of the raceway after grinding is shown in Fig. 7. As can be seen from Fig. 7, the roughness is between $0.6 \mu \mathrm{m}$ and $0.3 \mu \mathrm{m}$. With the increase of the speed of the grinding wheel, the roughness tends to decrease.

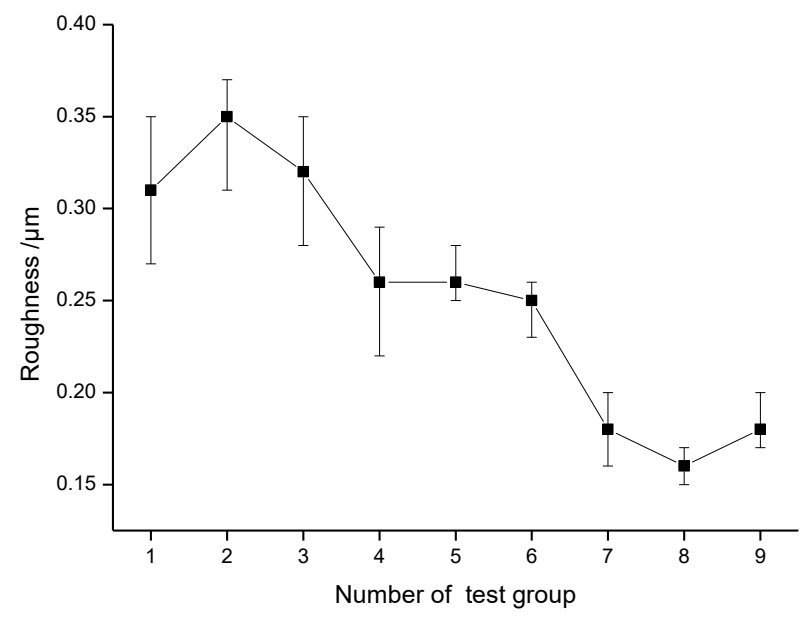

Fig. 7 Mean value of roughness

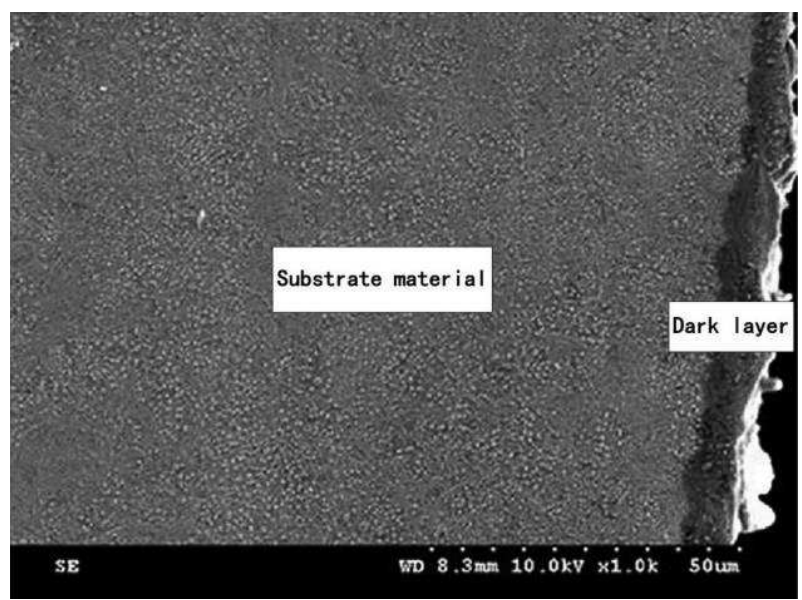

Fig. $\boldsymbol{8}$ Representative SEM micrograph showing the layer affected by grinding and the substrate

The roughness of the surface of the raceway was measured according to Form Talysurf PGI 1240.

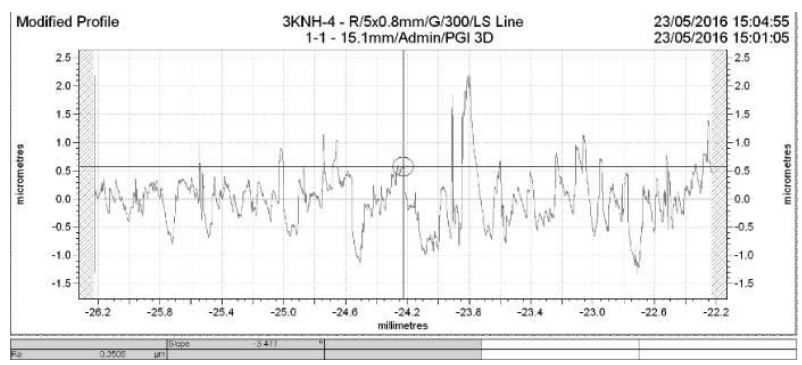

Fig. 9 Roughness measurement results

\section{Scatter calculation}

There are range, standard deviation, coefficient of variation and so on. In this paper, the most common standard deviation is used to characterize the residual stress scatter.

$$
\begin{gathered}
S=\sqrt{\frac{\sum\left(x_{i}-\bar{x}\right)^{2}}{n-1}} \\
\bar{x}=\frac{\sum x_{i}}{n}
\end{gathered}
$$

Where. $x_{i}$ is the test point data, $S$ is the standard deviation, $\bar{x}$ is the mean value, and $n$ is the total number of test points.

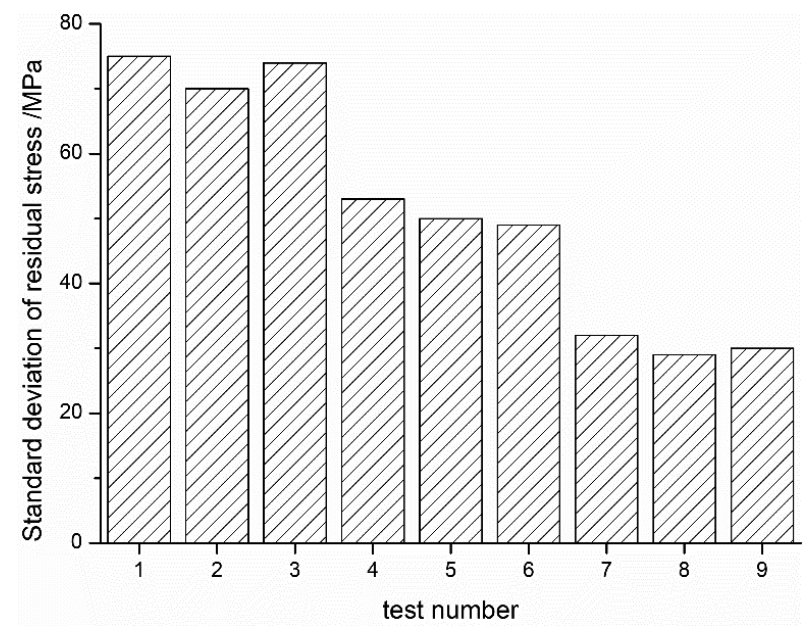

Fig. 10. Standard deviation of circumferential residual stress

It can be seen from Fig. 10 that the standard deviation of circumferential residual stress decreases with the increase of wheel speed. The standard deviation of circumferential residual stress of test group No. 1 3 is about $80 \mathrm{MPa}$, that of test group No. $4 \sim 6$ is about $60 \mathrm{MPa}$, and that of test group No. $7 \sim 9$ is about $30 \mathrm{MPa}$.

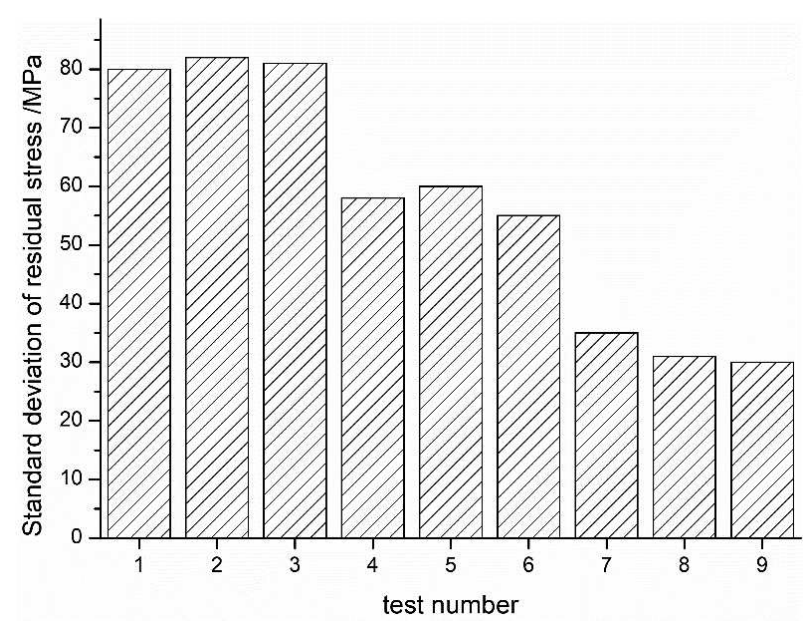

Fig. 11. Standard deviation of axial residual stress

It can be seen from Fig. 11 that the standard deviation of axial residual stress decreases with the increase of wheel speed. The standard deviation of circumferential residual stress of test group $1 \sim 3$ is about $75 \mathrm{Mpa}$, that of test group $4 \sim 6$ is about $50 \mathrm{MPa}$, and that of test group $7 \sim 9$ is about $30 \mathrm{MPa}$. 


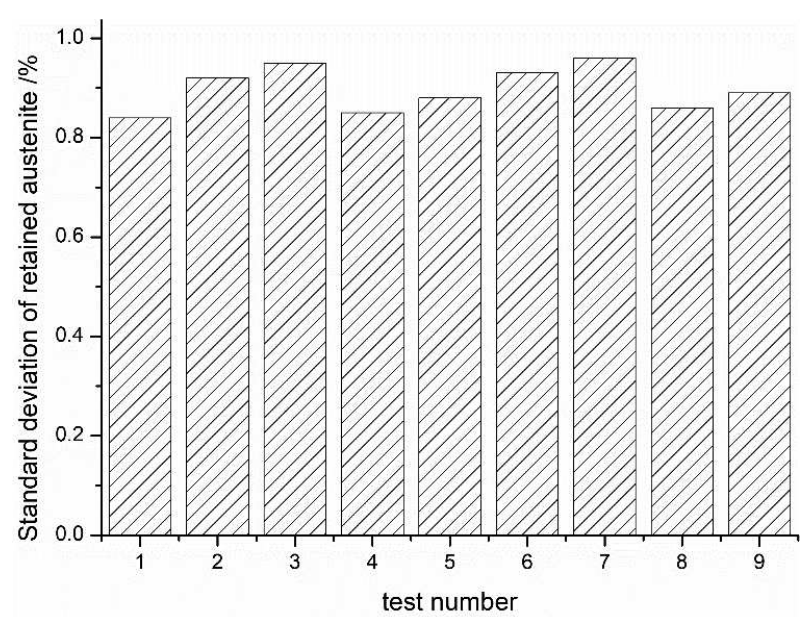

Fig. 12. Standard deviation of retained austenite

It can be seen from $\boldsymbol{F i g} .12$ that the standard deviation of retained austenite of test group No. 1 9 has little change and fluctuates around $0.9 \%$.

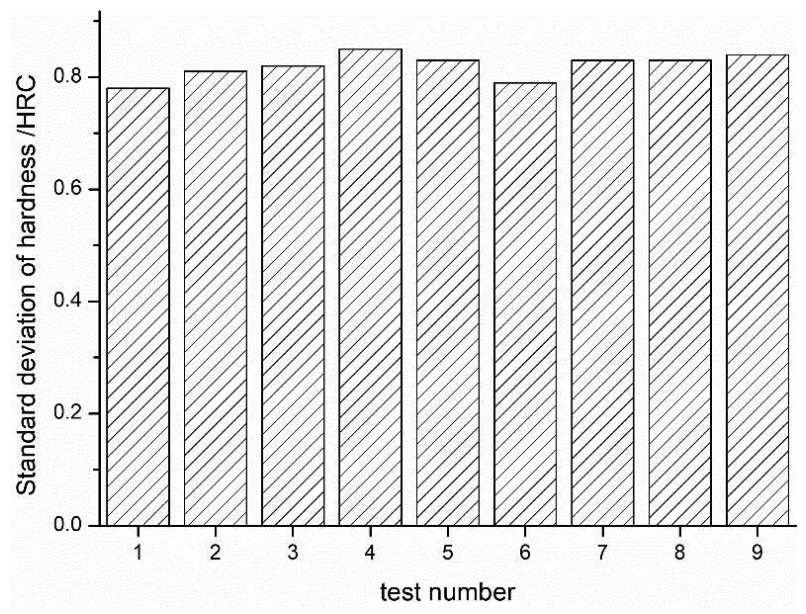

Fig. 13. Standard deviation of hardness

It can be seen from Fig. 13 that the standard deviation of hardness of test group No. 1 9 does not change much and fluctuates around 0.8HRC.

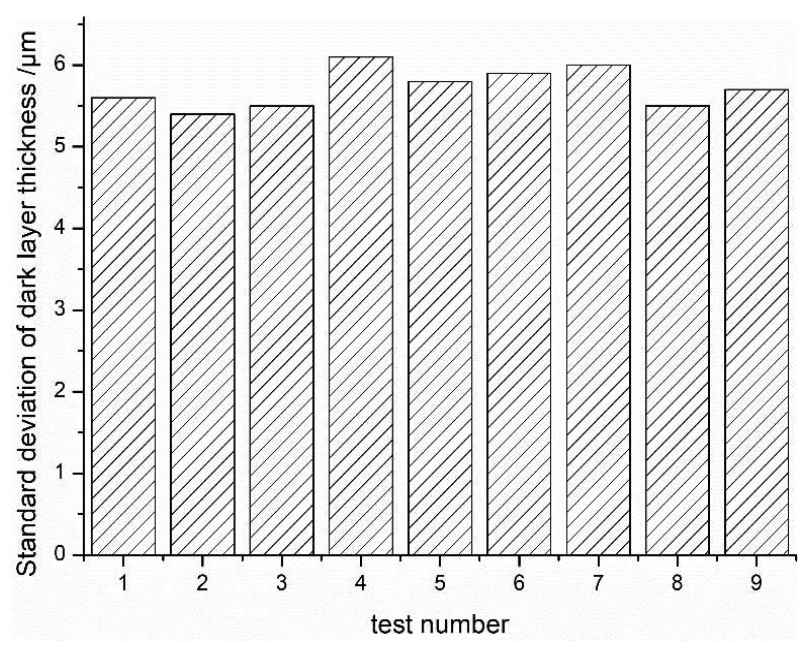

Fig. 14. Standard deviation of dark layer thickness
It can be seen from Fig. 14 that the standard deviation of dark layer thickness of test group No. 1 9 has little change and fluctuates around $5.5 \mu \mathrm{m}$

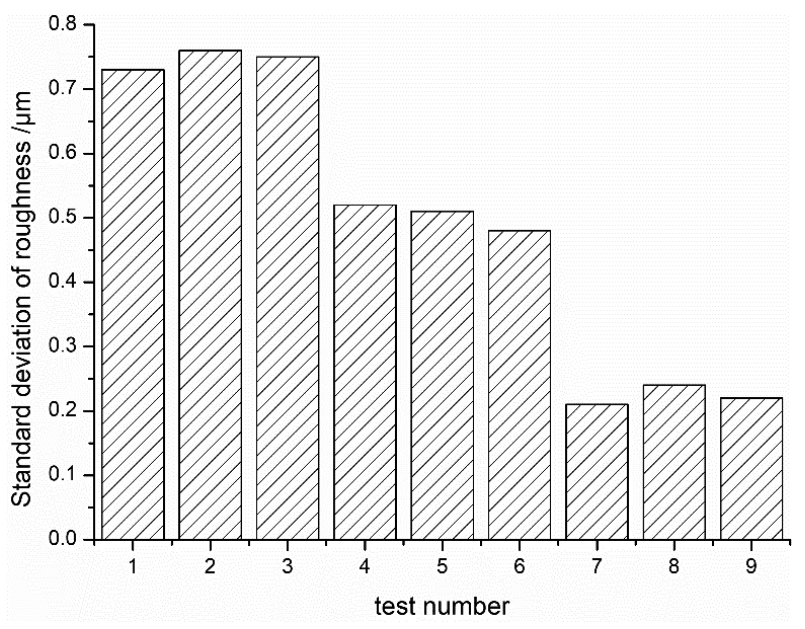

Fig. 15. Standard deviation of roughness

It can be seen from Fig. 15 that the standard deviation of roughness decreases with the increase of wheel speed. The standard deviation of roughness of test group 1-3 is about $0.75 \mu \mathrm{m}$, that of test group 4-6 is about $0.45 \mu \mathrm{m}$, and that of test group 7-9 is about $0.2 \mu \mathrm{m}$.

\section{Scatter analysis}

The standard deviation of all indexes of bearing raceway surface integrity can be divided into two groups: in the orthogonal test, the surface integrity indexes with little change of standard deviation can be regarded as the first group, including the content of retained austenite, hardness and thickness of dark layer. In the orthogonal test, the surface integrity index with obvious standard deviation change can be regarded as the second group, including circumferential residual stress, axial residual stress and roughness.

In the first group of surface integrity indexes, the standard deviation of retained austenite content in 9 groups of test results did not change much, floating around $0.9 \%$. When the content of retained austenite is $8 \% \sim 10 \%$, the fatigue life of bearing is the best. By analyzing the mean value of retained austenite and the standard deviation of retained austenite content, it can be seen that the standard deviation of retained austenite content does not affect the fatigue life of the bearing, that is to say, the standard deviation of retained austenite content in 9 groups of tests meets the requirements of bearing use. In the first group of surface integrity indexes, the hardness standard deviation of 9 groups of test results changed little, floating around 0.8HRC. When the hardness is 60HRC $\sim 65 \mathrm{HRC}$, the wear resistance of the bearing is the best. By analyzing the hardness mean value and combining the hardness standard deviation, it can be seen that the hardness 
standard deviation does not affect the wear resistance of the bearing, that is to say, the hardness standard deviation of 9 groups of tests meets the requirements of bearing use. In the first group of surface integrity indexes, the standard deviation of dark layer thickness in the 9 groups of test results has little change, floating around $5.5 \mu \mathrm{m}$. As long as there is no white layer, the thickness of the dark layer has no effect on the fatigue life of the bearing [9]. By analyzing the mean value of the thickness of the hidden layer and combining with the standard deviation of the thickness of the hidden layer, it can be seen that the standard deviation of the thickness of the hidden layer does not affect the fatigue life of the bearing, that is to say, the standard deviation of the thickness of the hidden layer in nine groups of tests meets the requirements of the bearing.

In the second group of surface integrity indexes, the standard deviation of residual stress in 9 groups of test results changed obviously. With the increase of wheel speed, the standard deviation of residual stress decreases. The residual compressive stress can improve the bearing fatigue life, and the residual tensile stress can reduce the fatigue life. On the premise of meeting the requirements of the mean value of residual stress, we hope that the standard deviation of residual stress is small, which can make the bearing fatigue life keep a good consistency. Considering the mean value of residual stress and the standard deviation of residual stress, it is found that the scatter of circumferential and axial residual stress in test group 4 $\sim 6$ meets the requirements of bearing application. In the results of test group 4-6, the mean value of axial residual stress and circumferential residual stress are both compressive stress, and the standard deviation of axial residual stress and circumferential residual stress are small. In the second group of surface integrity indexes, the roughness of 9 groups of test results changed significantly. With the increase of the wheel speed, the standard deviation of roughness decreases. Lower roughness can reduce the vibration and noise of bearing in service. On the premise of meeting the requirements of the mean roughness, we hope that the standard deviation of the roughness is better, which can improve the rotation accuracy of the bearing. At the same time, considering the mean and standard deviation of roughness, it is found that the scatter of roughness in the test group No. $7 \sim 9$ meets the requirements of bearing use. Among the results of test group 7-9, the mean value of roughness is the smallest, and the standard deviation of roughness is the smallest.

\section{Discussion}

In the concept of discrete degree of bearing raceway surface integrity, we need to put forward requirements for the mean value and standard deviation of each index of bearing raceway surface integrity. In order to better study the standard deviation of each index of bearing raceway surface integrity, the above has been divided into two groups according to the change trend of standard deviation. The purpose of studying the standard deviation of each index is also to meet the requirements of bearing use. In order to meet the requirements of standard deviation and mean value of all indexes, the grinding parameters used in test group $4 \sim 6$ are better grinding process. In the grinding process parameters of test group $4 \sim 6$, considering the grinding efficiency, the grinding parameters adopted by test group 5 are the best grinding process.

The test results show that the rotation speed of grinding wheel is the most important grinding parameter affecting the surface integrity of bearing raceway. With the increase of wheel speed, the scatter of grinding residual stress becomes smaller. This is because the number of grains increases in the unit time of highspeed grinding, homogenizing the grinding residual stress. With the increase of wheel speed, the scatter of grinding roughness becomes smaller. This is due to the increase of the number of grains per unit time in high-speed grinding, which homogenizes the grinding roughness. Therefore, high-speed grinding has homogenization effect and can homogenize the surface integrity index with obvious standard deviation change.

\section{Conclusion}

Considering the scatter of raceway surface integrity, the optimal grinding parameters of grinding orthogonal test are as follows: wheel speed $7000 \mathrm{R} / \mathrm{min}$, workpiece speed $150 \mathrm{R} / \mathrm{min}$, grinding depth $15 \mu \mathrm{m}$.

The indexes with obvious change of standard deviation include residual stress and roughness, while the indexes without obvious change of standard deviation include retained austenite content, hardness and thickness of grinding modified layer.

The rotation speed of grinding wheel is the main reason for the change of standard deviation. For the index with obvious standard deviation change, higher wheel speed has the effect of standard deviation homogenization. For the index of stable variation of standard deviation, higher wheel speed has no effect on standard deviation.

\section{Acknowledgement}

\section{We gratefully acknowledge the valuable coo- peration of Dr. Qian Jia and the members of her laboratory in preparing this Application note.}

\section{Reference}

[1] GUO, Y., LI, W., JAWAHIR, I. (2009). Surface integrity characterization and prediction in machining of hardened and difficult-to-machine 
alloys: a state-of-art research review and analysis[]. Machining Science and Technology, 13(4): 437470.

[2] YUAN, Z-J., HU, H-Z., KOBAYSHII, A. (1989). Surface integrity of grinding of bearing steel GCr15 with CBN wheels[]. CIRP annals, 38(1): 553-556.

[3] ABRAO, A. M., ASPINWALL, D, K. (1996). The surface integrity of turned and ground hardened bearing steel[]. Wear, 196(1-2): 279-284.

[4] KONIG, W., BERKTOLD, A., KOCH, K-F. (1993). Turning versus grinding-a comparison of surface integrity aspects and attainable accuracies[]. CIRP annals, 42(1): 39-43.

[5] LIU, C. R., YANG, X. (2001). The scatter of surface residual stresses produced by face-turning and grinding[J]. Machining science and techno$\log y, 5(1): 1-21$.

[6] GAO, E-W. (2008). Experimental study and numerical analysis of surface residual stress dispersion in precision grinding [D]. Shanghai: School of mechanical and power engineering, Shanghai Jiaotong University

[7] ZHANG, X-P., WANG, H-P., LU, G-H., et al. (2010). Experimental study on surface residual stress dispersion of bearing steel after sequential grinding [J]. Journal of mechanical engineering, (15): 89-94

[8] STANCEKOVA, D., RUDAWSKA, A., NESLUSAN, M., MRAZIK., J., JANOTA, M. (2019). The implementations of suitable cutting parameters by grinding of titanium VT9 with impact on surface integrity []. Manufacturing Technology, 19(5):855-859

[9] MAREK, M., NOVAK, M., ŠRAMHAUSER, K. (2019). The tmpact of changes in inFeed rate on surface integrity after chrome plate grinding by silicon carbide[J]. Manufacturing Technology, 19(2):284-291

[10] HARRIS, T A. (2001). Rolling bearing analysis[M]. John Wiley and sons, 2001.

[11] GUO, Y., SCHWACH, D. W. (2005). An experimental investigation of white layer on rolling contact fatigue using acoustic emission technique[]. International journal of fatigue, 27(9): 1051-1061.

[12] ALMEN, J. O., BLACK, P. H. (1963). Residual stresses and fatigue in metals[M]. McGraw-Hill

[13] SU, Y-T., LIN, M-H., LEE, M-S. (1993). The effects of surface irregularities on roller bearing vibrations[J]. Journal of sound and vibration, 165(3): 455-466. 